\title{
Repercussões do ensino a distância no processo de formação em enfermagem na Pandemia da COVID-19
}

\author{
Repercussions of remote learning in Nursing Education in the COVID-19 pandemic \\ Repercusiones del aprendizaje a distancia en el proceso de formación en enfermería en la pandemia \\ COVID-19
}

\author{
Ursula Silva Baptista Chaves \\ ORCID: https://orcid.org/0000-0003-2272-3624 \\ Universidade do Estado do Rio de Janeiro, Brasil \\ E-mail: ursulasbchaves@gmail.com \\ Carolina Cabral Pereira da Costa \\ ORCID: https://orcid.org/0000-0002-1345-0612 \\ Universidade do Estado do Rio de Janeiro, Brasil \\ E-mail: carolcuerj@hotmail.com \\ Norma Valéria Dantas de Oliveira Souza \\ ORCID: https://orcid.org/0000-0002-2936-3468 \\ Universidade do Estado do Rio de Janeiro, Brasil \\ E-mail: norval_souza@yahoo.com.br \\ Eloá Caneiro Carvalho \\ ORCID: https://orcid.org/0000-0002-1099-370X \\ Universidade do Estado do Rio de Janeiro, Brasil \\ E-mail: eloagrossi@uol.com.br \\ Samira Silva Santos Soares \\ ORCID: https://orcid.org/0000-0001-9133-7044 \\ Universidade Federal do Rio de Janeiro, Brasil \\ E-mail: samira_opg@hotmail.com \\ Patrícia Britto Ribeiro de Jesus \\ ORCID: https://orcid.org/0000-0003-4523-3740 \\ Universidade do Estado do Rio de Janeiro, Brasil \\ E-mail: patty_brj@hotmail.com \\ Helena Ferraz Gomes \\ ORCID: https://orcid.org/0000-0001-6089-6361 \\ Universidade do Estado do Rio de Janeiro, Brasil \\ E-mail: helenafg1@yahoo.com.br \\ Ellen Marcia Peres \\ ORCID: https://orcid.org/0000-0003-4262-6987 \\ Universidade do Estado do Rio de Janeiro, Brasil \\ E-mail: ellenperes@globo.com \\ Lívia Fajin de Mello \\ ORCID: https://orcid.org/0000-0002-5613-7976 \\ Universidade do Estado do Rio de Janeiro, Brasil \\ E-mail:liviafajin@gmail.com \\ Priscila Cristina da Silva Thiengo de Andrade \\ ORCID: https://orcid.org/0000-0003-0840-4838 \\ Universidade do Estado do Rio de Janeiro, Brasil \\ E-mail: profprithiengo@gmail.com \\ Cilene Bisagni \\ ORCID: https://orcid.org/0000-0001-7153-7495 \\ Centro Universitário Celso Lisboa, Brasil \\ E-mail: cilene.bisagni@celsolisboa.edu.br \\ Manoel Luís Cardoso Vieira \\ ORCID: https://orcid.org/0000-0003-1614-5848 \\ Universidade Federal do Rio de Janeiro, Brasil \\ E-mail: maneu.vieira@gmail.com
}

\section{Resumo}

Objetivo: refletir sobre o ensino à distância no processo de formação em enfermagem no contexto de pandemia da COVID-19. Método: artigo de reflexão que tem como eixo central a pandemia da COVID-19 e o ensino à distância $(\mathrm{EaD})$ na formação do enfermeiro, pautando-se em três seções teóricas: i) EaD: breve contextualização, vantagens e desvantagens ii) Formação de Enfermeiros mediante o ensino à distância: críticas, desafios e implicações iii) 
Repercussões da pandemia COVID-19 no processo de formação de enfermeiros. Resultados: a enfermagem tem como foco principal o cuidado, e a graduação em enfermagem busca a formação de profissionais que sejam capazes de realizar atividades de planejamento, organização, supervisão, além de implementar e executar cuidados voltados ao cliente e à comunidade. Apesar dos esforços para implementação do ensino remoto, em decorrência da pandemia, é possível perceber que no contexto da graduação em enfermagem, tal fato acarretará prejuízos ao processo ensinoaprendizagem. Considerações Finais: A formação graduada em enfermagem propõe-se a formar profissionais que sejam capazes de atuar nos serviços de saúde, o que pressupõe a necessidade da práxis para o desenvolvimento de habilidades técnicas, relacionais e gerenciais. Portanto, há limitações importantes a serem consideradas neste contexto pandêmico voltadas para formação do profissional enfermeiro.

Palavras-chave: Enfermagem; Educação em enfermagem; Ensino; Pandemias; Infecções por coronavirus.

\begin{abstract}
Objective: to reflect upon distance learning in the process of educational development in Nursing in the context of the COVID-19 pandemic. Methodology: a reflexive article which has as its central axis the COVID-19 pandemic and distance learning (EaD in Portuguese), and the educational development of nurses, focusing on three theoretical sections: i) Distance education: brief contextualisation, advantages and disadvantages, ii) The education of nurses in the context of distance education: criticism, challenges and implications, iii) Repercussions of the coronavirus pandemic in the educational process of nurses. Results: Nursing has as its main focus the caring of others and the Bachelor of Science in Nursing seeks to enable professionals to carry out planning, organising and supervising activities as well as implementing and providing clients and communities with special care. In spite of the effort to implement distance education, as a consequence of the pandemic, it is noticeable that in the context of the field of Nursing such fact leads to losses in the teaching-learning process. Concluding remarks: The Bachelor of Science in Nursing aims to assist professionals in being capable of performing duties in the healthcare industry, which presupposes the need of praxis for the development of technical, relational and managerial abilities. Therefore, there are considerable limits to be taken into consideration in this pandemic context as far as the educational development of nurses is concerned.
\end{abstract}

Keywords: Nursing; Nursing education; Teaching; Pandemics; Coronavirus infections.

\begin{abstract}
Resumen
Objetivo: reflexionar sobre la educación a distancia en el proceso de formación en enfermería en el contexto de la pandemia COVID-19. Método: artículo de reflexión que tiene la pandemia COVID-19 y la educación a distancia (EaD) como eje central en la formación de enfermeras, basado en tres apartados teóricos: i) EaD: contextualización breve, ventajas y desventajas ii) Formación de enfermeras a distancia aprendizaje: críticas, desafíos e implicaciones iii) Repercusiones de la pandemia COVID-19 en el proceso de formación de enfermeras. Resultados: la enfermería se enfoca principalmente en el cuidado, y el programa de pregrado en enfermería busca formar profesionales que sean capaces de realizar actividades de planificación, organización, supervisión, además de implementar y ejecutar cuidados dirigidos al cliente y la comunidad. A pesar de los esfuerzos para implementar la educación a distancia, debido a la pandemia, es posible darse cuenta de que en el contexto de la carrera de enfermería, este hecho provocará pérdidas en el proceso de enseñanza-aprendizaje. Consideraciones finales: La formación de posgrado en enfermería tiene como objetivo formar profesionales capaces de trabajar en los servicios de salud, lo que presupone la necesidad de praxis para el desarrollo de habilidades técnicas, relacionales y gerenciales. Por tanto, existen importantes limitaciones a considerar en este contexto pandémico orientadas a la formación del profesional de enfermería.
\end{abstract}

Palabras clave: Enfermería; Educación en enfermería; Enseñando; Pandemias; Infecciones por coronavírus.

\title{
1. Introdução
}

O ano de 2020 ficará marcado na história por uma grave crise sanitária mundial que tem mobilizado governos em todo o mundo. O novo coronavírus iniciou sua circulação em Wuhan, província da China (WHO, 2020) e, devido a sua acelerada disseminação por diversos países e continentes, a Organização Mundial de Saúde (OMS) decretou, em 11 de março de 2020 , estado de pandemia (OPAS, 2020).

De acordo com resumos científicos publicados pela OMS, a disseminação do Severe Acute Respiratory Syndrome (SARS-CoV-2), agente etiológico do Coronavírus Diasease (COVID-19), ocorre de pessoa a pessoa desde a fase présintomática, principalmente por meio de gotículas emitidas pela fala, tosse, espirro e contato físico próximo, em ambiente fechado por período superior a uma hora. Há evidência, também, de transmissão por aerossóis gerados durante procedimentos médicos e, também por objetos inanimados contaminados, os chamados fômites (McEnroe, 2020). 
Diante da inexistência de medicamentos antivirais específicos para combater a doença e da limitada disponibilidade de doses das vacinas para a imunização da população mundial, até o presente momento, os princípios de Florence Nightingale nunca estiveram tão contemporâneos. Nesta perspectiva, medidas como a higienização das mãos com água e sabão, fricção com álcool 70\%, higienização dos hospitais e dos ambientes domésticos, adoção de etiqueta respiratória, utilização de máscara de tecido em locais públicos, manutenção de ambiente limpo e arejado, além da prática do distanciamento social, evitando-se aglomerações, seguem imperativas para o controle da circulação do vírus e de suas novas variantes (McEnroe, 2020; OPAS, 2020; WHO, 2021).

Cabe ressaltar que a COVID-19 é uma infecção respiratória que evolui de formas diferentes em cada indivíduo, inclusive existem pessoas que mesmo infectadas não apresentam sintomatologia clínica, já as sintomáticas podem desenvolver a doença em sua forma leve, moderada ou grave e, até mesmo, evoluir para o óbito (OPAS, 2020). Até o dia até 02 de abril de 2021, a OMS registrou, em todo o mundo, 129.215 .179 casos confirmados e 2.820.098 mortes decorrentes da doença (WHO, 2021). No Brasil, o Ministério da Saúde divulgou através do Boletim Epidemiológico Especial nº52, referente à Semana Epidemiológica 08 de 2021, que até o dia 27 de fevereiro do ano corrente, 10.517.232 casos confirmados e 254.221 óbitos pela COVID-19 em território brasileiro (Brasil, 2021).

Diante deste cenário, reacendeu a discussão em relação ao Ensino à Distância (EAD) na formação do profissional de enfermagem. Assim, em situação emergencial, houve a necessidade de se recorrer ao ensino à distância, com vistas a dar prosseguimento ao processo formativo. Desde então, o Ministério da Educação vem publicando diversas portarias no Diário Oficial da União, sendo a mais atual a Portaria $N^{\circ} 544 / 20$, que autoriza as instituições de ensino superior realizarem as atividades letivas por meio de "recursos educacionais digitais, tecnologias de informação e comunicação ou outros meios convencionais", em substituição às aulas presenciais, até a data de 31 de dezembro de 2020 (Brasil, 2020, p.1).

Destaca-se que no texto, o documento responsabiliza as instituições quanto a disponibilização dos recursos necessários para que os discentes estejam aptos a acompanhar as atividades letivas, assim como, realizar as avaliações de conteúdo (Brasil, 2020).

Nesta lógica, como forma de continuar promovendo a aprendizagem e minimizar prejuízos na formação dos discentes, preservando a saúde de todos os envolvidos, instituições de ensino superior públicas e privadas se viram obrigadas a suspender as aulas presenciais e aderir ao formato de ensino remoto emergencial, cabendo, assim, ressaltar que as aulas nesse formato não são consideradas uma modalidade de ensino, apenas uma solução acessível para atender a necessidade em tempos de pandemia.

Desta forma, o ensino à distância, atualmente, tem sido considerado uma relevante estratégia para o processo formativo, já que rompe com paradigmas como a sincronia e a presença física de docentes e discentes em um mesmo local (Alves et al., 2015). Mesmo à distância, tem-se buscado adotar metodologias pedagógicas que proporcionem ao discente ocupar a posição de protagonista do seu processo de ensino-aprendizagem, rompendo-se com o modelo tradicional de ensino, estimulando-o a pensar soluções utilizando a problematização de situações reais (Souza et al., 2018).

Uma dessas possibilidades metodológicas que rompem com o ensino tradicional se inclina na metodologia conhecida como PBL - Aprendizagem baseada em problemas. Este método consiste em uma proposta pedagógica de ensino centrado no estudante e baseado na solução de problemas, conforme informa Silva, Dias, Souza e Lima (2019). De acordo com as autoras nesse modelo de metodologia de ensino, o estudante adquire ferramentas para desenvolver habilidades técnicas, cognitivas e atitudinais para a prática profissional o que corrobora diretamente na formação do enfermeiro.

Essa modalidade metodológica se classifica no Brasil como parte das metodologias ativas, estas que são abalizadas pelos princípios teóricos de Paulo Freire, objetivando-se a formação de um profissional autônomo, crítico-reflexivo, com 
responsabilidades que estejam em consonância com o mundo real e que seja capaz de resolver os problemas a partir de conhecimentos prévios da realidade onde vive (Jesus et al., 2020).

Portanto, cabe ressaltar que a formação em enfermagem tem por objetivo preparar profissionais para o cuidar, deve ser direcionada para a atuação nos serviços de saúde do Sistema Único de Saúde (SUS), embasada pelos seus princípios e diretrizes e fundamentada no conceito ampliado de saúde. Para isso, faz-se necessário que esta formação seja voltada para o campo de trabalho, a fim de promover a integração entre o ensino e os serviços de saúde, e que o futuro enfermeiro possa se familiarizar com as políticas de saúde vigentes, a atuação multiprofissional e transdisciplinar que visam a atenção integral à saúde da população (Winters et al., 2016).

Tal questão é descrita nas Diretrizes Curriculares Nacionais (DCN), vigentes a partir de 2001, onde a mesma descreve que a formação do enfermeiro tem por objetivo dotar o profissional dos conhecimentos requeridos para o exercício de competências, enquanto capacidade de articular e mobilizar conhecimentos, habilidades e atitudes, colocando-os em ação para resolver problemas e enfrentar situações de imprevisibilidade; além das habilidades gerais: atenção à saúde, tomada de decisões, comunicação, liderança, administração, gerenciamento e educação permanente (Brasil, 2001).

Assim, na DCN, fica evidente o entendimento da necessidade em articular e mobilizar habilidades, estas que para o aluno em formação se traduzem nas habilidades práticas que são aquelas treinadas em laboratório de habilidades, de simulação realística, de anatomia e diversos outros tipos os quais compõem significativamente nas competências que deverão ser desenvolvidas. Porém com o advento da pandemia, a falta das atividades práticas poderá traduzir em impactos futuros significativos na formação do enfermeiro.

Nesta acepção, o presente artigo tem como objetivo refletir sobre o ensino à distância no processo de formação em enfermagem no contexto de pandemia da COVID-19. Compreende-se que a contribuição deste estudo é destacar a necessidade de ampliação das discussões voltadas para o processo de formação do enfermeiro de maneira remota, nas redes públicas e privadas, em meio à pandemia da COVID-19, e as possíveis repercussões que esta mudança possa gerar no futuro.

\section{Metodologia}

Trata-se de um artigo de reflexão que tem como eixo central a pandemia da COVID-19 e o ensino à distância na formação do enfermeiro. Esta reflexão foi desenvolvida pautando-se em três seções teóricas, as quais se complementam e viabilizaram o aprofundamento de discussões acerca da temática proposta.

As seções teóricas foram intituladas da seguinte forma: i) EAD: breve contextualização, vantagens e desvantagens; ii) Formação de enfermeiros mediante o ensino à distância: críticas, desafios e implicações; iii) Repercussões da pandemia COVID-19 no processo de formação de enfermeiros.

\section{Resultados e Discussão}

\section{EAD: breve contextualização, vantagens e desvantagens}

O percurso do EaD no Brasil perpassa por diferentes gerações, tendo seu início motivado pela necessidade de capacitação profissional da população frente ao processo de industrialização (Silva et al., 2016).

Inicialmente, essa modalidade ocorria por meio do envio de material por correspondência, porém com a expansão das mídias de comunicação de massa, diversos cursos passaram a ser oferecidos utilizando-se o rádio, a televisão e, até mesmo, vídeos, com o fito de ampliar o acesso da classe trabalhadora à educação (Fernandes et al., 2019).

Com o advento da Internet inaugura-se um novo momento e os cursos à distância se expandem, ganhando grandes proporções. Graças a utilização dessa tecnologia, distâncias foram encurtadas, possibilitando que pessoas de diferentes 
localidades estivessem conectadas em tempo real, além de facilitar a troca de informações e o envio de materiais a partir de apenas um clique (Fernandes et al., 2019).

Cabe destacar que, no Brasil, a expansão dos cursos à distância ocorreu no final da década de 1980, juntamente com a evolução das tecnologias educacionais e com a possibilidade de maior acesso ao computador e à internet, elementos que vêm se intensificando nos últimos anos (Marin et al., 2017).

Neste contexto, o EaD passou a ser uma modalidade de ensino prevista legalmente a partir da implementação da Lei $n^{\circ}$ 9.394/96 Lei de Diretrizes e Bases da Educação Nacional (LDB) (Brasil, 1996) e regulamentada pelo decreto ${ }^{\circ}$ 5.622/2005 (Brasil, 2005), revogado, posteriormente, pelo decreto ${ }^{\circ}$ 9.057/2017, que traz a seguinte definição de educação à distância:

modalidade educacional na qual a mediação didático-pedagógica nos processos de ensino e aprendizagem ocorra com a utilização de meios e tecnologias de informação e comunicação, com pessoal qualificado, com políticas de acesso, com acompanhamento e avaliação compatíveis, entre outros, e desenvolva atividades educativas por estudantes e profissionais da educação que estejam em lugares e tempos diversos. (Brasil, 2017, p.1)

O decreto supracitado autoriza a oferta de cursos de formação superior - graduação e pós-graduação latu sensu - no formato $\mathrm{EaD}$, desde que estejam credenciados junto ao Ministério da Educação e ocorram de acordo com a legislação em vigor. Nos casos em que o projeto pedagógico prevê atividades presenciais do tipo: aulas em laboratório, prática profissional, tutoria, estágio e defesa de trabalho de conclusão de curso, essas atividades devem ser desenvolvidas na sede da Instituição de Ensino Superior, em polos de ensino à distância mantidos pela instituição ou em ambientes profissionais (Brasil, 2017).

Em um país de grandes dimensões e marcado por relevante desigualdade social, como é o caso do Brasil, um percentual significativo da população não tem acesso à educação pela dificuldade de locomoção, falta de recursos para custear alimentação e meios de transporte, além da impossibilidade de estudar em horário instituído pelas instituições de ensino (Tavares, 2018) devido a elevadas jornadas de trabalho. O ensino à distância constitui-se, então, um recurso que democratiza o acesso à educação, possibilitando que uma maior parte da população possa se profissionalizar, impactando positivamente nas condições de empregabilidade.

Além disso, tem como condição sine qua non que o discente assuma a responsabilidade com seu processo de aprendizagem e desenvolva seu próprio método de estudo para que, através do autogerenciamento, consiga cumprir os prazos de entrega das tarefas (Antunes et al., 2019; Fernandes et al., 2019; Tavares, 2018), o que estimula a autonomia. Outro ponto positivo é que tal formato requer do aluno a leitura de maior quantidade de conteúdo, o que possibilita o treinamento de habilidades como interpretação de texto e concentração.

Nesta perspectiva, o EaD tem se mostrado um importante aliado na difusão de conhecimentos, por possibilitar que um maior número de pessoas tenha acesso aos conteúdos disponibilizados virtualmente, permitindo que o usuário estude em qualquer local e no horário que estiver disponível (Fernandes et al., 2019; Tavares, 2018).

Observa-se como desvantagem a diminuta interação entre discente-discente e docente-discente, pois na maioria das vezes o diálogo ocorre através dos fóruns de respostas, havendo um intervalo de tempo para que as dúvidas sejam esclarecidas, tornando o processo moroso. A impossibilidade de realizar atividades em grupo priva o aluno de experienciar momentos de debate, negociação e articulação, fundamentais para o trabalho em equipe.

Por se tratar de um formato muito teórico, há uma perda no que diz respeito a articulação de teoria e prática, haja visto que os momentos presenciais não são exigidos por lei em todos os cursos e, quando obrigatórios, esses ocorrem em menor quantidade, comparado aos cursos presenciais. 


\section{Formação de enfermeiros mediante o ensino à distância: críticas, desafios e implicações}

A partir da implementação do Sistema Único de Saúde (SUS), fez-se necessário a formação de profissionais capacitados para atuar de acordo com as políticas e serviços do modelo assistencial vigente. Diante de tal demanda, a graduação em enfermagem, que outrora objetivava a formação de profissionais para trabalhar com a doença no contexto hospitalar, precisou ser revista e vem sofrendo reformulações, a fim de capacitar pessoas detentoras de pensamento reflexivo, crítico, orientados por princípios éticos, habilitados para atuarem de acordo com as necessidades de saúde da população (Peres et al., 2018).

Desta maneira, o currículo teve que ser formulado articulando-se o tripé ensino, pesquisa e extensão, de forma a proporcionar uma formação integral e pautada no rigor científico, adotando-se metodologias que tenham o discente como centro do processo de ensino aprendizagem, no qual o professor ocupe a posição de mediador, estimulando e aguçando a curiosidade do discente para que este aprenda a aprender (Brasil, 2001).

No contexto do formato EaD, ficou autorizada a inserção de disciplinas no Projeto Pedagógico dos Cursos de Graduação em Enfermagem (PPC), desde que em observância às Diretrizes Curriculares Nacionais (DCN) do Curso de Graduação em Enfermagem que "definem os princípios, fundamentos, condições e procedimentos da formação de enfermeiros" (Brasil, 2001, p.1)

No entanto, enfatiza-se a importância da associação de teoria e prática, desde o início da formação, para que o formando desenvolva habilidades que o capacite a pensar criticamente sobre a realidade social onde está inserido e, com isso, possa buscar soluções. Espera-se, então, que sejam formados enfermeiros com perfil generalista, humanista, que embasem sua prática em princípios éticos e atuem promovendo ações que visem a proteção, promoção, prevenção e reabilitação do indivíduo, família e coletividade (Brasil, 2001).

No contexto da graduação em enfermagem, os órgãos representativos da categoria, Conselho Federal de Enfermagem (COFEN) e Associação Brasileira de Enfermagem (ABEn), preocupados em preservar a qualidade da formação profissional, tecem duras críticas ao ensino de enfermagem no formato $\mathrm{EaD}$, se posicionando radicalmente contra tal modalidade por entenderem que a formação integralmente à distância torna-se frágil e, consequentemente, pode impactar na qualidade da assistência prestada à população, gerando, por exemplo, aumento de erros por imprudência, negligência ou imperícia (Sanes et al., 2020).

Ademais, a crítica também se refere à adoção da modalidade apenas como forma de baratear o custo da formação para a instituição, sem priorizar o rigor metodológico. Preocupam-se com a infraestrutura dos polos de ensino à distância, no que diz respeito à qualidade e adequação dos laboratórios, assim como a mediação do momento presencial por tutores que possuem a função de aplicar o conteúdo disponibilizado pela matriz, tornando desnecessário o papel do docente (Sanes et al., 2020).

Porém, benefícios são apontados na incorporação de disciplinas no formato $\mathrm{EaD}$ ao longo da graduação em enfermagem. Na era digital, as Tecnologias Digitais de Informação e Comunicação (TDIC) permitem que conteúdos sejam armazenados em ambientes virtuais, aulas sejam transmitidas e experiências sejam trocadas entre discentes e docentes. Esta forma de desenvolver aprendizagem requer do discente a gestão do próprio processo educativo, estimulando habilidades como organização e autonomia (Silva et al., 2016; Tavares et al., 2018).

Estudo realizado em uma Instituição Federal de Ensino Superior de Minas Gerais, Brasil, sobre a percepção de estudantes de enfermagem a respeito da utilização do $\mathrm{EaD}$, demonstra que os discentes apontaram como ponto positivo a possibilidade de realizar disciplinas oferecidas em outros campus da mesma universidade e, também, em outras Instituições de Ensino Superior (IES), na área da saúde, da enfermagem e até mesmo de outras áreas, a exemplo do curso de inglês (Silva et al., 2016). 
Ressalta-se que o mercado de trabalho do século XXI, cada vez mais exigente, prima pela certificação (Frota et al., 2020). Na área da saúde, a força de trabalho continua sendo humana, porém com intensa incorporação tecnológica, demandando dos trabalhadores constante atualização de modo a acompanhar as aceleradas transformações do setor (Frota et al., 2020; Tavares et al., 2018).

Entretanto, faz-se mister considerar que a enfermagem tem como foco principal o cuidado e, por esta razão, o curso de graduação em enfermagem visa a formação de profissionais que sejam capazes de realizar atividades de planejamento, organização, supervisão, além de implementar e executar cuidados de enfermagem de forma direta ao cliente e à comunidade. Assim, aspirando garantir à população assistência embasada nos princípios e diretrizes do sistema de saúde vigente e com foco na humanização (Silva et al., 2016), destaca-se que apesar de todos os esforços para implementar nos cursos de enfermagem o ensino à distância ou ensino remoto, este apresenta limitações que podem comprometer a formação do profissional que necessita desenvolver habilidades, competências e atitudes frente ao processo de cuidar.

\section{Repercussões da pandemia COVID-19 no processo de formação de enfermeiros}

A história mundial é marcada por surtos de doenças que surgem de tempos em tempos, dizimam parte da população e a humanidade as enfrenta de acordo com os recursos disponíveis em cada época. O ano de 2020 está sendo marcado pela pior pandemia da atualidade, ocasionada pelo vírus SARS-CoV-2 que causa a COVID-19 (WHO, 2020b) e a sociedade do século XXI tem utilizado o aparato tecnológico existente na tentativa de minimizar seus impactos.

Ressalta-se que o Brasil já enfrentava dificuldades na área da educação, tendo a pandemia agravado a desigualdade de acesso a esse direito fundamental, que é a base de uma sociedade igualitária, justa e desenvolvida (Unesco, 2020).

A pandemia da COVID-19 fortaleceu as reflexões sobre o processo de formação nas IES públicas e privadas. Nesse sentido, fez emergir de forma emergencial uma série de discussões a respeito do processo e da necessidade de reinventar novas formas de ensino, mantendo-se a qualidade no processo de formação. Isto porque foram necessárias medidas para o enfrentamento dos desafios gerados pela pandemia também no campo da educação.

O Ministério da Educação intentando dar continuidade ao ano letivo de 2020, autorizou que cursos, de uma forma geral, utilizassem o ensino mediado por tecnologias, ensino remoto ou EAD, de modo excepcional, para viabilizar o processo de ensino e aprendizagem. A partir disso, surgiram diversos desafios tanto para os gestores das instituições de ensino públicas e privadas, quanto para os docentes, discentes e seus familiares.

Com vistas a possibilitar o ensino, pesquisa e extensão durante a pandemia, IES se viram na urgência de implementar o ensino remoto. Para atender tal demanda, mantendo a mesma qualidade do formato presencial, fez-se indispensável grande mobilização entre profissionais de diferentes áreas, que enfrentaram longos períodos de reuniões para discutir desde aspectos referentes à tecnologia para a implantação de ambientes virtuais, até questões como a metodologia utilizada, carga horária de discentes e docentes, critérios de avaliação, presença e interatividade dos atores envolvidos (Bezerra et al., 2020; Souza et al., 2020).

Foi preciso, com isso, replanejar o ano letivo, as estratégias pedagógicas, imersas e subsidiadas pelo uso da internet, a fim de reduzir os impactos na aprendizagem oriundos do ensino presencial, tendo em vista que os currículos de grande parte das instituições de ensino não foram criados para serem aplicados remotamente.

Nesta perspectiva, os docentes tiveram que se reinventar nas suas maneiras de ministrar aulas e avaliar estudantes, já que a maior parte não estava acostumada nem preparada para o ensino à distância, o qual pressupõe o afastamento geográfico de docentes e discentes. Assim como os estudantes, pois também tiveram que se adaptar a este "novo normal".

À vista disto, se percebe rotineiramente as dificuldades enfrentadas neste novo contexto formativo, já que a formação de bacharel em enfermagem exige atividades gerenciais e de assistência à saúde, que pressupõem contato presencial, empatia e 
interação para o processo de formação em serviço. Nesta ótica, verifica-se que é um grande desafio a utilização de um ensino à distância, pois apesar do emprego de tecnologias inovadoras e de metodologias ativas, não se tem verdadeiramente a operacionalização do contato humano.

Uma outra questão importante que se tem visto na prática do ensino remoto durante esta pandemia e que, de alguma maneira, impacta no processo formativo é a dificuldade de acesso aos ambientes virtuais. Tal situação ocorre por inexperiência na utilização desses ambientes e pela carência de recursos, como internet rápida ou recursos de informática avançados, criando obstáculos para que os discentes possam participar ativamente das aulas e das demais estratégias utilizadas, como o acesso aos materiais das aulas.

Assim, as TDIC constituem-se como a espinha dorsal para a viabilização do ensino exclusivamente à distância, trazendo à tona questões relacionadas ao acesso e à utilização dos meios digitais por docentes e discentes. Porém, as mesmas não chegam para todos os grupos da sociedade, devido à assimetria na distribuição de recursos materiais e financeiros, inaugurando mais uma forma de segregar pessoas e grupos (Knop, 2017).

Considera-se que para obtenção de benefícios na utilização das tecnologias digitais não basta apenas ter à disposição um aparelho conectado à internet, é mister conciliar fatores relacionados à infraestrutura, como qualidade do equipamento e capacidade da banda larga utilizada para promover um acesso adequado à rede, com os saberes necessários para a utilização das ferramentas de informática (Knop, 2017).

Portanto, de modo a garantir a equidade educacional, faz-se relevante que governo e universidades firmem parcerias com empresas de telecomunicação, de desenvolvimento e fabricação de softwares e produtos tecnológicos para que seja garantido ao estudante o acesso ao conteúdo disponibilizado na rede. Sanada a questão do acesso, a preocupação recai sobre a habilitação para utilização do ambiente virtual. Estudos anteriores destacam a relevância do treinamento de docentes e discentes, anterior ao início da utilização da plataforma digital, objetivando possibilitar o engajamento do discente (Antunes et al., 2019; Souza et al., 2020), sobretudo nesse grande desafio de se manter estudando em um momento adverso como uma pandemia, em um formato exclusivamente à distância de maneira compulsória.

Outro ponto que impacta na motivação é a utilização de uma estratégia pedagógica atrativa, utilizando-se formas variadas na apresentação das disciplinas intercalando conteúdos textuais com atividades em grupo, ao vivo, onde o professor possa valer-se de recursos inovadores para estimular a pesquisa, o debate, enfim, a troca de conhecimento. Ressalta-se que manter a relação entre professor e alunos é fundamental na mediação pedagógica em ambientes virtuais (Antunes et al., 2019).

Salienta-se que a graduação em enfermagem intenta formar profissionais com competências e habilidades para a prática do cuidado da pessoa e coletividades, assim como para enfrentar as adversidades e os agravos à saúde da população. Para isso, prima-se por um currículo que articule teoria e prática desde o início do curso com o fito de aproximar o estudante da realidade das ações e serviços de saúde ofertados pelo SUS (Brasil, 2001). Neste sentido, tem-se uma grande perda com o EaD, pela limitação em relação à realização de atividades práticas e teórico-práticas.

O Conselho Nacional de Saúde (CNS), em 01 de julho de 2020, emitiu a recomendação $n^{\circ}$ 048, posicionando-se contrário à realização de práticas profissionais de estágio e laboratório no formato EAD e/ou remoto nos cursos da área da saúde. Tal recomendação fundamenta-se no entendimento de que a formação de profissionais da saúde exige presencialidade para que o formando em contato com profissionais e usuários, vivenciando situações reais, possa desenvolver capacidades de escuta, empatia, comunicação, acolhimento, além de propiciar o exercício das habilidades técnicas relativas à cada área do saber (Brasil 2020b).

O documento sinaliza que as TDIC devem ser utilizadas como dispositivos pedagógicos complementares ao processo de ensino e aprendizagem e não como forma de substituir o ensino presencial (Brasil 2020b), pretendendo com isso 
garantir formação de qualidade aos profissionais de saúde, tanto em situações de emergência sanitária, como é o caso da atual crise pandêmica, quanto em condições habituais.

Em relação à inserção de acadêmicos nos serviços de saúde durante a pandemia da COVID-19, o CNS enfatiza a necessidade de que esta atividade ocorra sob supervisão pedagógica e chama à responsabilidade os serviços e instituições de ensino no sentido de providenciar condições de segurança aos estudantes e preceptores. Essas condições seguras dizem respeito ao fornecimento de EPI (máscaras N95, aventais, óculos, protetores faciais e luvas) de modo que esteja garantida a integridade física e psicossocial dos estagiários e professores (Brasil 2020b).

Entretanto, apesar de extremamente importante, é uma situação delicada, que se constitui inclusive como uma barreira para inserção de acadêmicos nos serviços de saúde, tendo em vista que, por vezes, não há recursos suficientes nem para a equipe do próprio serviço, quanto mais em quantidade e qualidade suficientes para uso de estudantes e dos professores supervisores.

Ademais, a pandemia se soma às fragilidades já encontradas no processo de trabalho da enfermagem, agravando situações como as péssimas condições de trabalho, sobrecarga laboral levando ao desgaste físico e mental dos profissionais e a ausência de EPI em número suficiente para a proteção do trabalhador (Miranda et al., 2020).

No entanto, cabe destacar que o fato de os estudantes de enfermagem se manterem afastados dos serviços de saúde gera repercussões importantes que podem impactar diretamente no desenvolvimento de habilidades, tomadas de decisões e competências. Então, no que se refere ao ensino de enfermagem, salienta-se que a discussão vai além do fato de não se estar presencialmente em uma sala de aula, mas principalmente pela impossibilidade de serem realizadas atividades teórico-práticas e práticas.

A limitação da realização das atividades práticas pelos graduandos de enfermagem neste momento, notoriamente gera lacunas no processo formativo e exige dos docentes o estabelecimento de estratégias que permitam o desenvolvimento das habilidades técnicas sem colocar em risco a saúde dos professores e estudantes. Tudo isso gera um sentimento de medo e incertezas na formação em enfermagem, exatamente no ano de 2020, o qual foi tão esperado por marcar o bicentenário do nascimento de Florence Nightingale e que foi declarado pela OMS como o "Ano Internacional da Enfermagem".

\section{Considerações Finais}

É inegável que está sendo feito um esforço considerável no sentido de viabilizar estratégias voltadas para redução de danos no processo de ensino-aprendizagem, na tentativa de se deixar o processo menos frágil, enquanto perdurar esta situação de pandemia.

É incontestável, ainda, que a mudança para o ensino remoto fez emergir a capacidade inovadora dos docentes, que precisaram se adaptar à nova metodologia de forma repentina, ao implementarem ferramentas diferenciadas, se reinventando no processo educacional.

Todavia, não se pode perder de vista que a graduação em enfermagem forma profissionais para atuar no serviço de saúde, o que pressupõe a necessidade da interação teoria e prática, para o desenvolvimento de habilidades técnicas, relacionais e gerenciais. E neste sentido, especialmente para a graduação em enfermagem há limitações importantes a serem consideradas neste contexto pandêmico, voltadas a formação do enfermeiro.

Desta forma, compreendendo a relevância do tema frente à pandemia causada pelo COVID-19, ressalta-se que esta temática não se finda por meio deste estudo e muitas observações poderão emergir dessa reflexão. Considerando-se assim, de grande relevância que mais estudos sejam realizados enfatizando esse tema. 


\section{Referências}

Alves, V.L.S.; Bohomol, E. \& Cunha, I.C.K. (2015). Educação de pós-graduação em enfermagem à distância: avaliação sob a perspectiva dos discentes. Acta paul enferm, 28(2), 139-145. doi: http://dx.doi: 10.1590/ 1982-0194201500024

Antunes, F. R.; Alves, F. C.; Pina-Oliveira, A. A.; Apostolico, M. R. \& Puggina, A. C. (2019). Motivação de alunos da área da saúde em disciplinas totalmente a distância: influência socioeconômica. Cogitare enferm, 24(e60243), 1-13. doi: http://dx.doi.org/10.5380/ce.v24i0.60243

Bezerra, K. P.; Costa, K. F. L.; Oliveira, L. C.; Fernandes, A. C. L.; Carvalho, F. P. B. \& Nelson, I. C. A. S. R. (2020). Ensino remoto em universidades públicas estaduais: o futuro que se faz presente. Research, Society and Development, 9(9), 1-17. doi: http://dx.doi.org/10.33448/rsd-v9i9.7226

Brasil. Conselho Nacional de Saúde. (2020). Recomendação $n^{o}$ 048, de 01 de julho de 2020. Recomenda ao Ministério da Educação, que observe o Parecer Técnico n ${ }^{\circ}$ 162/2020, no que diz respeito a estágios e práticas na área da saúde durante a pandemia de COVID-19.

Brasil. Ministério da Educação. Conselho Nacional de Educação. Câmara de Educação Superior. (2001). Resolução CNE/CES No 3, de 7 de novembro de 2001. Institui as Diretrizes Curriculares Nacionais do Curso de Graduação em Enfermagem.

Brasil. Ministério da Educação. (2005). Decreto n ${ }^{\circ}$ 5.622, de 19 de dezembro de 2005. Regulamenta a Educação a Distância.

Brasil. Ministério da Educação. (2017). Decreto n ${ }^{\circ}$ 9.057, de 25 de maio de 2017. Regulamenta a Educação a Distância.

Brasil. Ministério da Educação. (1996). Lei $n^{\circ}$ 9.394, de 20 de dezembro de 1996. Estabelece as diretrizes e bases da educação nacional.

Brasil. Ministério da Educação (2020). Portaria N 544 de 16 de junho de 2020. Brasília, 2020.

Brasil. Ministério da Saúde. (2021). Boletim Epidemiológico Especial 52 - doença pelo coronavírus - COVID-19.

Fernandes, S. M.; Henn, L. G. \& Kist, L. B. (2019). O ensino a distância no Brasil: alguns apontamentos. Research, Society and Development, 9(1), 1-24. doi: http://dx.doi.org/10.33448/rsd-v9i1.1551

Frota, M. A.; Wermelinger, M. C. M. W.; Vieira, L. J. E. S., Ximenes Neto, F. R. G., Queiroz, R. S. M. \& Amorim, R. F. (2020). Mapeando a formação do enfermeiro no Brasil: desafios para atuação em cenários complexos e globalizados. Ciência \& Saúde Coletiva, 25(1), 25-35. doi:10.1590/141381232020251.27672019

Knop, M. F. T. (2017). Exclusão digital, diferenças no acesso e uso de tecnologias de informação e comunicação: questões conceituais, metodológicas e empíricas. Caderno Eletrônico de Ciências Sociais, 5(2), 39-58, doi: https://doi.org/10.24305/cadecs.v5i2.2017.19437

Jesus, P.B.R.; Vieira, M.L.C; Silva, W.P.; Costa, C.C.P.; Bisagni, C.; Villarinho, D.R.L. Em tempos de COVID-19 e ensino EaD: a criatividade da monitoria acadêmica de um curso de enfermagem. (2020). In: Educação a Distância na era COVID: possibilidades, limitações, desafios e perspectivas. Organizadora Solange Aparecida de Souza Monteiro. - Ponta Grossa - PR: Atena.

Marin, M. J. S.; Nascimento, E. N.; Tonhom, S. F. R.; Alves, S. B. A. D.; Girotto, M. A.; Otani, M. A. P. \& Silva, L. C. P. (2017). Formação na modalidade a distância pela Universidade Aberta do SUS: Estudo qualitativo sobre o impacto do curso na prática profissional. Rev bras educ méd, 41(2), 201-209. doi: http://dx.doi.org/10.1590/1981-52712015v41n2RB20160003

McEnroe, N. (2020). Celebrating Florence Nightingale's bicentenary. The Lancet, 395 (10245), 1475-1478. doi: https://doi.org/10.1016/S0140$6736(20) 30992-2$

Miranda, F. M. A.; Santana, L. L.; Pizzolato, A. C \& Saquis, L. M. M. (2020). Condições de trabalho e o impacto na saúde dos profissionais de enfermagem frente a COVID-19. Cogitare enferm, 25(e7270). doi: http://dx.doi.org/10.5380/ce.v25i0.72702)

Organização Pan-Americana da Saúde - OPAS. (2020). Modes of transmission of virus causing COVID-19: implications for infection prevention and control (IPC) precaution recommendations.

Organização Pan-Americana da Saúde - OPAS. 2020.

Peres, C.R.F.B.; Marin, M. J. S.; Soriano, E. C. L. \& Ferreira, M. L. S. M. (2018). Um olhar dialético para as mudanças curriculares na formação do enfermeiro. Rev. Esc. Enferm. USP, 52(e03397), 1-8. doi: http://dx.doi.org/10.1590/S1980-220X2017038003397

Sanes, M. S.; Neves, F. B.; Pereira, L. E. M.; Ramos, F. R. S.; Brehmer, L. C. F.; Vargas, M. A. O. \& Martini, J.G. (2020). Educação a distância, não! Produção de sentidos dos discursos de entidades representativas da enfermagem. Rev Bras Enferm, 73(5), 1-11. doi: http://dx.doi.org/10.1590/0034-71672019-0465

Silva, L. T. C.; Diniz, F. A.; Gontijo, T. L.; Machado, R. M. \& Cavalcante, R. B. (2016). Percepções de estudantes de enfermagem sobre educação a distância. Ciencia y enfermeira, 22(2), 129-139.

Silva, E.S.; Dias, B.J.C.; Souza, J.L.; Lima, M.S. (2019). Aprendizagem baseada em problema aplicada no ensino de urgência e emergência na enfermagem: um relato de experiência. Braz J Hea Rev, Curitiba, 2 (4), 2525-2529.

Souza, C. J.; Guerra, T. R. B.; Carvalho, D. S.; Jesus, R. V. L.; Costa, L. H. O.; Issobe, M. K.; Vieira, H. L. S.; Santos, D. A. \& Zamba, C. F. S. (2020). As interfaces da (re) invenção do ensino na graduação em enfermagem em tempo de COVID-19. Research, Society and Development, 9(7), 1-19. doi: http://dx.doi.org/10.33448/rsd-v9i7.4190

Souza, E. F. D.; Silva, A. G. \& Silva, A. I. L. F. (2018). Active methodologies for graduation in nursing: focus on the health care of older adults. Rev Bras Enferm, 71(suppl 2), 920-4, 2018. doi: http://dx.doi.org/10.1590/0034-7167-2017-0150 
Research, Society and Development, v. 10, n. 5, e27510514702, 2021

(CC BY 4.0) | ISSN 2525-3409 | DOI: http://dx.doi.org/10.33448/rsd-v10i5.14702

Tavares, A. P. C.; Leite, B. S.; Silveira, I. A.; Santos, T. D.; Brito, W. A. P. \& Camacho, A. C. L. F. (2018). Analysis of Brazilian publications on distance education in nursing: integrative review. Rev Bras Enferm, 71(1), 214-222. doi: http://dx.doi.org/10.1590/0034-7167-2016-0454

UNESCO - Organização das Nações Unidas para Educação, Ciência e Cultura. (2020). Webinário promovido pelo Movimento Nacional ODS Santa Catarina discute caminhos para minimizar os efeitos da pandemia na educação.

Winters, J. R. F.; Prado, M. L. \& Heidemann, I. T. S. B. (2016). A formação em enfermagem orientada aos princípios do Sistema Único de Saúde: percepção dos formandos. Esc Anna Nery, 20(2), 248-253. doi: 10.5935/1414-8145.20160033

World Health Organization - WHO. Coronavirus. 2020.

World Health Organization - WHO. (2021). Coronavirus (COVID-19) Dashboard.

World Health Organization - WHO. (2020). Coronavirus disease (COVID-19) outbreak: rights,roles and responsibilities of health workers, including key considerations for occupational safety and health. 